\title{
Just the facts: how to assess for cerebral venous thrombosis
}

\author{
Ariel Hendin ${ }^{1}\left[\right.$ [ Lana Castellucci ${ }^{2} \cdot$ Hans Rosenberg ${ }^{1}$ \\ Received: 9 June 2021 / Accepted: 5 August 2021 / Published online: 24 August 2021 \\ (C) The Author(s), under exclusive licence to Canadian Association of Emergency Physicians (CAEP)/ Association Canadienne de Médecine d'Urgence \\ (ACMU) 2021
}

Keywords Thrombosis $\cdot$ COVID $\cdot$ Stroke

\section{Clinical scenario}

A 38-year-old woman is brought to the emergency department (ED) with a severe headache that began 36 hours ago. She has a past medical history of ulcerative colitis and her medications include sulfasalazine as well as an oral contraceptive pill. Her partner called 911 because she had a first witnessed generalized tonic-clonic seizure at home. She is post-ictal but recovering when you see her, and on exam, she is globally weak. Vital signs, point-of-care-glucose, and basic bloodwork are normal. You order a plain CT head which does not show any acute abnormalities. The patient's partner arrives and is quite worried that she received the AstraZeneca ${ }^{\circledR}$ COVID vaccine 14 days ago. You consider if there is any further workup you need to do at this point.

\section{Clinical questions}

\section{What is Cerebral Venous Thrombosis (CVT) and how common is it?}

CVT occurs when blood clots form in the cerebral cortical veins or dural venous sinuses. This leads to increased intracranial pressure, cerebral edema, and infarction of brain tissue, which can subsequently hemorrhage. CVT accounts for $0.5-1 \%$ of all stroke diagnoses. While this is a rare disease, affecting approximately five patients per million yearly, a delayed diagnosis of CVT can lead to significant morbidity

Ariel Hendin

ahendin@toh.ca

1 Department of Emergency Medicine, University of Ottawa, 1053 Carling Avenue, Ottawa, ON K1Y4E9, Canada

2 Department of Medicine, University of Ottawa, Ottawa Hospital Research Institute, Ottawa, ON, Canada and mortality. CVT is more common among younger patients, with a median age of 33-37 years, and the majority are female (in a $3: 1$ ratio).

CVT can manifest in multiple ways. Most patients (90\%) report persistent headache, but some will present with seizure, or with focal neurological deficits that will vary based on the region of venous occlusion. A minority of patients with CVT will present with confusion as a predominant symptom if they have developed cerebral edema $[1,2]$.

\section{What are the risk factors associated with CVT?}

CVT is a thrombotic disease of the venous system of the brain, and $75-85 \%$ of patients carry at least one typical risk factor for thromboembolism. Risk factors in order of decreasing frequency include oral contraceptive use or hormone replacement therapy, pregnancy, postpartum state, acquired/hereditary thrombophilia, and local trauma or inflammation, such as a head and neck infection or surgery [1]. Systemic diseases, such as cancer, inflammatory bowel disease, and vasculitides, are also associated with CVT.

\section{What is vaccine-induced immune thrombotic thrombocytopenia (VITT)?}

Recently, CVT has been documented as a rare, serious thrombotic complication of adenovirus vector COVID-19 vaccines. Cases of arterial and venous thrombosis, including CVT, have been reported among recipients of adenoviral COVID vaccines who develop VITT (Astrazeneca ${ }^{\circledR}$ or Johnson \& Johnson ${ }^{\circledR}$ ). The pathophysiology of VITT is a prothrombotic disorder that rarely develops after vaccination, in which antibodies against platelet factor IV develop. These antibodies, which are also seen in heparin-induced thrombocytopenia, then cause platelet activation, aggregation, and arterial or venous clots. The trigger in VITT appears to be vaccination itself (not heparin exposure), and most cases are 
reported within 4-28 days of vaccination. The incidence of VITT ranges from 1 in 26,000 to 1 in 100,000. There has not been any association reported between typical venous thromboembolism risk factors and increased risk of VITT [3].

\section{How is CVT diagnosed in the emergency department?}

No lab test can rule out the diagnosis of CVT. While a D-dimer assay is highly sensitive for pulmonary embolism and deep venous thrombosis, its use in CVT is less well defined. The D-dimer had a sensitivity of $89 \%$ in a recent meta-analysis of patients with CVT [4], but false negative results are seen particularly in patients with prolonged symptom duration (greater than 1 week) or with headache alone in the absence of other findings. This means that physicians in low-resource or rural settings without access to advanced imaging modalities will have to consider transfer of patients for definitive exclusion or confirmation of diagnosis.

Despite the limited utility of blood tests in CVT, plain computed tomography (CT) head is helpful as a first test. Non-contrast CT may show abnormalities consistent with CVT in up to $1 / 3$ of cases, including a hyperdense cortical vein or dural sinus, or foci of intracranial hemorrhage. However, the majority of non-contrast CT scans are normal in patients with CVT, and further testing must be pursued in a patient with high clinical suspicion [2]. Magnetic Resonance Venogram (MRV) is the gold standard for diagnosis. The sensitivity of CT venogram (CTV), based on small studies, is nearly as good as MRV (and likely more accessible in the ED) [4]. Classic findings on CTV include venous sinus rim enhancement and a central filling defect. In patients with a high level of suspicion for CVT after a nondiagnostic CTV, MRV should be considered.

If there is a specific concern for CVT in the context of recent adenoviral vector COVID vaccine, the first test should be a complete blood count. VITT is much less likely with a platelet count $>150 \times 10^{9} / \mathrm{L}$. Alternative diagnoses (including CVT unrelated to VITT, in a patient with clinical features or risk factors) should be considered [3]. Additional investigations for VITT diagnosis should be discussed with a Thrombosis expert.

\section{What are the key management steps in the treatment of CTV?}

All CVT patients, including those with intracerebral hemorrhage, should be started on low molecular weight heparin unless contraindicated due to renal insufficiency or if rapid reversal is necessary for surgical intervention. These latter patients should receive intravenous unfractionated heparin. Long-term management typically consists of warfarin, as there is limited evidence for direct oral anticoagulants, but these decisions are made with Thrombosis consultation.

Patients with increased intracranial pressure causing headache should receive analgesia and antiemetics. Patients with severe intracranial hypertension with signs of impending herniation on imaging should be referred for decompressive surgery. Antiepileptic medications should be started for CVT patients presenting with seizure. Steroids should not be used unless indicated for an accompanying inflammatory condition. Thrombolytics and endovascular therapy are not recommended [1, 2, 4, 5].

For patients with VITT and associated CVT, treatment involves avoiding heparin-based anticoagulants and platelet transfusions, and management should be discussed with a Thrombosis expert [3]. If VITT is suspected post-COVID vaccine, this should also be reported to the local public health agency.

Supplementary Information The online version contains supplementary material available at https://doi.org/10.1007/s43678-021-00194-9.

\section{Declarations}

Conflict of interest No conflicts of interest to declare on the part of any of the authors.

\section{References}

1. Ulivi L, Squitieri M, Cohen H, Cowley P, Werring DJ. Cerebral venous thrombosis: a practical guide. Pract Neurol. 2020;20(5):356-67.

2. Saposnik G, Barinagarrementeria F, Brown RD, et al. Diagnosis and management of cerebral venous thrombosis: a statement for healthcare professionals from the American Heart Association/ American Stroke Association. Stroke. 2011;42(4):1158-92.

3. Pai M, Chan B, Stall NM, et al. Vaccine-induced immune thrombotic thrombocytopenia (VITT) following adenovirus vector COVID-19 vaccination. 2021:1-9. https://covid19-sciencetable. $\mathrm{ca} /$ sciencebrief/vaccine-induced-immune-thrombotic-thrombocyt openia-vitt-followingadenovirus-vector-covid-19-vaccination/. Accessed 5 June 2021.

4. Ferro JM, Bousser MG, Canhão P, et al. European Stroke Organization guideline for the diagnosis and treatment of cerebral venous thrombosis-endorsed by the European Academy of Neurology. Eur J Neurol. 2017;24(10):1203-13.

5. Coutinho J, de Bruijn SF, de Veber G, Stam J. Anticoagulation for cerebral venous sinus thrombosis. Cochrane Database Syst Rev. 2011. 